\title{
La centralidad del derecho al cuidado en la crisis del COVID-19 en América Latina. Oportunidades en riesgo( $\left.{ }^{*}\right)$
}

\author{
The centrality of the Right to care in the Covid-19 crisis in Latin América. \\ Opportunities at risk
}

\author{
Laura Cecilia Pautassi $\left.{ }^{(* *}\right)$ \\ Universidad de Buenos Aires (Buenos Aires, Argentina)
}

Resumen: La pandemia del Covid-19 produjo un cambio de escenario en materia de derechos humanos que resulta imprescindible atender en toda su dimensión. En América Latina, la pandemia irrumpe en un momento en que los Estados ya estaban con una limitada capacidad de respuesta desde las politicas públicas. Las medidas adoptadas establecieron restricciones de circulación y dejaron al descubierto la injusta configuración del cuidado al interior de los hogares y a nivel social. Por cuidado, se entiende a todas aquellas actividades indispensables para satisfacer las necesidades básicas de la existencia y reproducción de las personas, brindándoles elementos físicos y simbólicos que les permiten vivir en sociedad.

En el presente artículo se analiza el alcance del reconocimiento del cuidado como trabajo, pero particularmente como derecho humano, (el derecho a cuidar, a ser cuidado y al autocuidado), a partir del relevamiento de estudios teóricos y empíricos desarrollados en América Latina. El objetivo es presentar el marco conceptual y evidencia empírica preexistente a la crisis del Covid-19 respecto al cuidado, para identificar la falta de su distribución social y la manera en que se concentra en las mujeres en desmedro de su autonomía. La hipótesis que guía el análisis es que hasta que no se adopten políticas universales, transversales, con presupuestos regulares y en base a un enfoque de género y de derechos, no se alcanzarán respuestas eficientes a la pandemia. El argumento que introduce el artículo, situado en un recorrido por algunos de los países de América Latina y de las recomendaciones de los órganos de protección de derechos humanos, es que, a pesar de la alusión a la universalidad, las políticas terminan focalizando por condición de vulnerabilidad sin promover acciones integrales, absolutamente necesarias durante la crisis, pero para diseñar la salida a la misma.

Palabras claves: Derecho al Cuidado - Covid-19 - América Latina - Enfoque de Derechos Humanos - Políticas Públicas

\begin{abstract}
The COVID-19 pandemic has brought about a change of scenery in the field of human rights that must be addressed in all its dimensions. In Latin America the pandemic erupted in a moment when States were already challenged in their regulations and response capacity to COVID-19. The recent measures adopted to prevent the pandemic first and foremost prescribe the restriction of movement and mandatory preventive social isolation. This brings to light the configuration of care within households and the unfair social organization around it. Care is usually understood as any activity that is essential in satisfying the basic needs for human existence and reproduction, providing the physical and symbolic elements that allow humans to live in society.
\end{abstract}

$\left.{ }^{*}\right) \quad$ Nota del Editor: Este artículo fue recibido el 20 de agosto de 2020 y su publicación fue aprobada el 25 de septiembre de 2020.

${ }^{* *}$ Doctora de la Universidad de Buenos Aires, Área Derecho Social. Profesora Adjunta regular, Facultad de Derecho (UBA) Directora Grupo Interdisciplinario Derechos Sociales y Políticas Públicas (www.dspp.com.ar) y del Programa Género y Derecho. ORCID: https://orcid.org/0000-0002-7393-9006. Correo electrónico: laurapautassi@derecho.uba.ar 
This paper analyzes the scope of the recognition of care as work but particularly as a human right, (the right to care, to be cared for and to self-care), based on the survey of theoretical and empirical studies developed in Latin America. The objective is to present the conceptual framework and empirical evidence pre-existing to the Covid 19 crisis regarding care, to identify the lack of its social distribution and the way in which it focuses on women to the detriment of their autonomy. The hypothesis guiding the analysis is that until universal, cross-cutting policies are adopted, with regular budgets and based on a gender and rights approach, efficient responses to the pandemic will not be achieved. The argument that the article introduces (set in a tour of some of the Latin American countries and the recommendations of the human rights protection bodies) is that despite the allusion to universality, the policies end up targeting due to vulnerability without promoting comprehensive actions, absolutely necessary during the crisis, but to design the way out.

Keywords: Care rights - Covid-19 - Latin America - Human Rights approach - Public policies

\section{Introducción: la irrupción del Covid-19}

Hace meses que no podemos hablar, pensar, sentir, estudiar o trabajar sin que el (o la) Covid-19 aparezca en sus múltiples manifestaciones. Nos atraviesa, nos paraliza, afecta el aire y los pulmones, pero también a la economía, la política, a la justicia y pone en evidencia una multiplicidad de situaciones de riesgo y de alto dramatismo. Los planes y proyecciones para esta segunda década del siglo en curso, quedaron atravesados por una de las crisis más severas de las que hemos conocido. Sin embargo, la pandemia desnudó una serie de situaciones preexistentes que interpelan respecto de las desigualdades estructurales y de la degradación de las condiciones de vida de millones de personas. Entre otras particularidades, la pandemia se ha caracterizado por la asombrosa y rápida propagación, junto con las graves consecuencias que acarrea, no sólo respecto a las vidas que ha cobrado sino precisamente por el desconcierto que produce y porque irrumpe en medio de otras crisis generalizadas.

En América Latina, la pandemia se presenta en un momento en que los Estados ya se encontraban con una limitada capacidad de respuesta económica, con crisis institucionales, políticas y sociales recurrentes y con una interpelación respecto de los límites de las políticas públicas para afrontar estas situaciones. La evidencia empírica disponible para la región muestra que durante el período 2010-2019 la tasa de crecimiento del PIB en América Latina disminuyó del $6 \%$ al $0,2 \%$; sin embargo, el período de menor crecimiento desde la década de 1950 ocurrió entre 2014-2019 (0,4\%), y para el 2020 se espera una contracción de la actividad económica en $5,3 \%$. Las estimaciones respecto a los indicadores socioeconómicos advierten que la tasa de pobreza se incrementará en 4,4 puntos porcentuales y la pobreza extrema 2,6 puntos porcentuales con respecto a 2019, lo que implicaría alcanzar al $34,7 \%$ y $13 \%$ respectivamente, con impactos también respecto del aumento de la desigualdad (CEPAL-OIT, 2020, p. 5). Una variedad de factores como el endeudamiento externo, la pobreza, desempleo e informalidad laboral, la baja y deteriorada cobertura en salud y educación, las movilidades humanas y las múltiples manifestaciones de violencias, particularmente la violencia de género, han agudizado las desigualdades, especialmente la injusta división sexual del trabajo y del cuidado.

Este agravamiento de las situaciones ya señaladas, integraban la agenda de derechos humanos previa y quedaron reflejadas en la reacción inmediata de los sistemas de protección internacional y regional, que incluyen numerosas declaraciones, estándares y recomendaciones por parte de los comités de los Pactos y Tratados Internacionales, de los expertos independientes y de órganos de fiscalización y control, entre otros ${ }^{(1)}$. Por ejemplo, la Comisión Interamericana de Derechos Humanos, además de sus comunicados y advertencias a los Estados sobre las medidas a adoptar respetuosas de derechos humanos, conformó la SACROI-COVID 19 (Sala de

(1) Solo a modo de ejemplo, se destacan las recomendaciones del experto independiente para la deuda externa con enfoque de género y reconocimiento especial de la economía del cuidado, https://www.ohchr.org/sp/NewsEvents/Pages/DisplayNews. aspx?News|D=25732\&LangID=s; el Comité de Expertas de Violencia de la Convención de Belem do Pará y la Comisión Interamericana de Mujeres (CIM-OEA) han desarrollado numerosos estudios y comunicados recomendando a los Estados las respuesta a las violencias, http://www.oas.org/es/cim/COVID-19.asp. Por parte del Comité CEDAW (https://www.ohchr. org/_layouts/15/WopiFrame.aspx?sourcedoc=/Documents/HRBodies/CEDAW/Statements/CEDAW_statement_COVID-19_final doc\&action=default\&DefaultttemOpen=1) y así en cada Comité, experto/a o relator/a especial de Naciones Unidas. 
coordinación y respuesta oportuna e integrada ante la crisis del COVID-19) con el objetivo de agilizar las respuestas y monitoreo de acciones de protección de derechos humanos, identificar situaciones de emergencia y promover medidas cautelares y soluciones urgentes.

En ese contexto, los derechos, sean civiles y políticos (DCP) o económicos, sociales, culturales y ambientales (DESCA) evidencian su universalidad, integralidad e interdependencia, precisamente debido a las situaciones de riesgo que la pandemia masivamente expone. El debate sobre restricciones constitucionales y libertades individuales se da en el mismo momento que los sectores bajo informalidad laboral no pueden acceder a un ingreso monetario, donde los poderes públicos tienen que readecuar su estrategia para garantizar el funcionamiento mínimo en torno a garantías de derechos, en medio del abuso de las fuerzas policiales y de seguridad(2).

Por su parte, la salud se encuentra en profundo riesgo, el acceso al agua, la vivienda y la infraestructura básica están altamente comprometidas en todas las metrópolis latinoamericanas, a lo que se suma que instituciones centrales como las escuelas y servicios públicos dejaron de funcionar y la violencia doméstica creció exponencialmente, sin que sea posible dar respuestas de manera simultánea y en las particularidades de cada caso. La pandemia no solo afecta a las personas más vulnerables, sino que atraviesa a toda la sociedad y paradojalmente a toda la humanidad.

A su vez, las recomendaciones y el accionar público se concentra en los cuidados. La centralidad del cuidado y de los cuidados (remunerados y no remunerados) se puso en evidencia prácticamente desde el mismo momento de declaración de la crisis sanitaria. De manera asombrosa, una actividad absolutamente indispensable para garantizar la sostenibilidad de la vida, históricamente invisibilizada y desvalorizada, emerge como la principal herramienta global para prevenir y transitar la pandemia. Todas las medidas adoptadas para la prevención, que establecen centralmente la restricción de circulación, aislamiento social obligatorio, cuarentena o medidas similares como el toque de queda, sacan a la luz la relevancia de la configuración de los cuidados al interior de los hogares. Sin embargo, el hecho de adoptar a los cuidados como elementos centrales para transitar la pandemia, no significa que se haya comprendido su centralidad y mucho menos que se haya indagado su configuración al interior de los hogares. Al igual que el esperado descubrimiento de la vacuna, las situaciones de cuidado esperan que alguien las resuelva, sin que se piense en cómo las resolvemos colectivamente.

Tampoco ha corrido mejor suerte el cuidado remunerado y todas las actividades vinculadas a los cuidados institucionalizados, en especial del sector salud y los denominados servicios esenciales en los diferentes países de la región. En el caso de quienes trabajan en la salud, transitan un derrotero de ser el recurso humano central de la crisis, destacando el carácter esencial y heroico de las trabajadoras y trabajadores, con aplausos y manifestaciones masivas de aprobación casi en paralelo con el repudio por los riesgos que pueden significar como agentes portadores de la "salud" y del "virus" al mismo tiempo, y con consiguiente ajuste y reducción salarial. Así la profundidad de la crisis no resiste la advertencia de Fraser (2016, p. 112) respecto a que "ninguna sociedad que sistemáticamente debilite su reproducción social logra perdurar mucho", identificando una nueva forma de capitalismo que socava las formas de reproducción social y afecta la sostenibilidad de la sociedad en su conjunto.

Estas situaciones ponen de manifiesto que la naturalización del cuidado como una responsabilidad femenina genera impactos negativos para la sociedad en general y para las mujeres en particular. Así las mujeres son quienes se encuentran más expuestas a la enfermedad por dedicarse al cuidado de la salud $-70 \%$ de los trabajos en salud a nivel mundial están a cargo de mujeres (Organización Internacional del Trabajo, 2019) - y durante el aislamiento obligatorio se encuentran sobrecargadas por nuevas demandas de cuidados de sus familiares. A ellos se adiciona, que en muchos casos han sufrido un recorte salarial o en otros directamente la cuarentena les ha significado la pérdida de trabajos remunerados, como en el caso del servicio doméstico o en cuidados interpersonales. Y a ellos se suma la presencia de múltiples violencias, en particular la violencia doméstica que ocurre al interior de las familias.

De esta forma, la pandemia nos confronta con las múltiples situaciones de desigualdad prexistentes que afectan el ejercicio de derechos humanos, en éste el continente más desigual de la tierra. En este artículo, analizo la consideración del cuidado como derecho humano, que incluye el derecho a cuidar, a ser cuidado y al autocuidado (Pautassi, 2007),

(2) Al respecto, ver los trabajos contenidos en Bohoslavsky (2020). 
a partir del relevamiento de estudios teóricos y empíricos desarrollados en América Latina. El objetivo es presentar el marco conceptual y evidencia empírica preexistente a la crisis del Covid-19 respecto al cuidado, para identificar la falta de su distribución social y la manera en que se concentra en las mujeres en desmedro de su autonomía. La hipótesis que guía el análisis es que hasta tanto no se adopten políticas universales, transversales, con presupuestos regulares y en base a un enfoque de género y de derechos, no se alcanzarán respuestas eficientes a la pandemia. El argumento que introduce el artículo, situado en un recorrido por algunos de los países de América Latina y de las recomendaciones de los órganos de protección de derechos humanos, es que pesar de la alusión a la universalidad, las políticas terminan focalizando por condición de vulnerabilidad sin promover acciones integrales, absolutamente necesarias durante la crisis pero para diseñar la salida a la misma.

Debido a la simultaneidad de la pandemia, y las consiguientes dificultades para la recolección de evidencia empírica, el análisis se realiza en base a fuentes secundarias y las respuestas de los sistemas de protección internacional para América Latina. En las conclusiones, y siempre desde un abordaje de derechos humanos, presento algunas alertas y escenarios posibles de la retórica de la "nueva normalidad" que llevan a cuestionar el efectivo alcance de las soluciones coyunturales sobre el marco de desigualdades estructurales, enfatizando la necesaria consideración del enfoque de género y de derechos humanos.

\section{El cuidado es un derecho humano}

El cuidado y los cuidados incluyen innumerables actividades que comprenden la alimentación, la salud, la salud mental, educación, vivienda, como también el desarrollo de habilidades cognitivas, físicas o emocionales (England et al., 2002). Es el trabajo invisible y cotidiano de cuidado el que permite la sostenibilidad de la vida, en una doble dimensión: (i) física, porque permite las actividades concretas vinculadas con la atención del cuerpo de las personas y sus necesidades de alimentación, salud, higiene personal, descanso; y (ii) simbólica, porque el cuidado involucra un componente afectivo y emocional, que hacen al bienestar de las personas. Si bien todas las personas necesitan de cuidados a lo largo de la vida, aquellas que son dependientes ya sea por encontrarse en los extremos de la vida (niñez, personas mayores) o por otras razones (por situaciones derivadas de enfermedades 0 personas con discapacidad que requieren apoyos especiales para el ejercicio de su autonomía) requieren de una mayor cantidad de cuidados o de cuidados especiales. Es decir, la característica principal es que "atraviesa" todos los órdenes de la vida y se inscribe en la construcción de nuestra subjetividad: está presente y se requiere a lo largo de nuestras vidas, en un tránsito permanente como demandantes a proveedores de cuidado en edades centrales y otra vez a demandantes, y así durante todo el ciclo vital requerimos de cuidados, prestados por otros $u$ otras, en algunos casos de modo permanente (personas con discapacidad o con enfermedades de larga duración) o autoprovistos, pero siempre presentes (Pautassi, 2019).

El cuidado, además de ser un trabajo intenso que debe ser valorado, es un derecho humano: "el derecho a cuidar, a ser cuidado y al autocuidado" (Pautassi, 2007) que se encuentra incorporado en Pactos y Tratados internacionales e integra el corpus de derechos humanos. En tanto derecho, impone obligaciones que deben ser cumplidas en el marco de la definición de políticas universales, transversales, con presupuestos regulares y en base a un enfoque de género, en interdependencia con el conjunto de derechos económicos, sociales y culturales (DESC) y civiles y políticos (DCP).

Cabe aclarar que el campo del Derecho no ha sido ajeno al cuidado, aunque originariamente se concentraba en la legislación dirigida a las familias en el marco del Derecho Civil con regulaciones que contemplaban los intereses del individuo sobre el de los familiares (Marco Navarro, 2009) y al individualizar se nubla la identificación de las responsabilidades de cuidado asignadas a las mujeres y que se distribuyen de manera inequitativa al interior del hogar. De manera relativamente reciente la transformación hacia el derecho de las familias ha permitido la incorporación del enfoque de género y de diversidades sexuales, pero en general circunscripto al ámbito privado sin incorporar su dimensión social.

El otro campo de regulación histórico es el derecho al trabajo, a partir de medidas de conciliación trabajo-familia con importantes sesgos de género, ya que en general regulan ciertos mecanismos como las licencias, infraestructura y transferencias monetarias organizadas a partir de los sistemas de seguridad social (asignaciones familiares). Los sesgos se manifiestan en las escasas disposiciones que incorporan a los varones, como también la normativa garantiza las condiciones para que las trabajadoras 
asalariadas formales puedan desempeñar sus tareas, perpetrando la naturalización del cuidado en las mujeres. Valga como ejemplo, que las licencias por nacimiento de hija o hijo para los varones cuando están reconocidas oscilan entre 2 a 5 días en muchos de los países de América Latina (Pautassi et al., 2004). Por otra parte, la consideración de la mujer asociada siempre en función de una posición -madre, trabajadora, esposa, pobre-marca las formas de organización y de desarrollo de los sistemas de políticas sociales regionales.

Sin embargo, ni las regulaciones laborales ni la doctrina jurídica ha reconocido derechos propios de las mujeres a cargo del cuidado, ya que persiste su acceso al sistema en función de derechos derivados - por el trabajo o por el vínculo legal con un trabajador- sin un reconocimiento integro de su condición de persona (Marco Navarro \& Pautassi, 2020). De allí la relevancia de la conceptualización del cuidado como derecho humano de cada persona, con total independencia de cualquier condición o posición que ocupe, y que no se concentre solo en las mujeres.

Este reconocimiento que incorpora estándares de universalidad marca el inicio de una transformación, ya que el cuidado y las obligaciones que genera para el Estado van a desplazarse del ámbito de las medidas de conciliación trabajofamilia presentes en el mundo del empleo hacia las políticas públicas.

En este marco, y de la conjunción de los trabajos teóricos y empíricos junto con el diálogo entre la academia feminista, los gobiernos y los organismos especializados de Naciones Unidas en América Latina se fue construyendo una agenda de cuidado "autóctona u originaria" que impulsó la promoción del reconocimiento del cuidado como derecho humano. Si bien no se encontraba formalizado ni denominado como derecho al cuidado, sí estaba incorporado en su alcance en los principales Pactos y Tratados Internacionales suscritos por los países de la región, como también en el corpus interpretativo de derechos humanos.

El hecho de haber cristalizado, a partir del enfoque de derechos humanos, que el cuidado es un derecho humano posibilitó, en primer lugar, centrarlo en la persona, desvinculada de otras condiciones de acceso, como en el caso del trabajo asalariado formal. En segundo lugar, al ser un derecho humano y activar una serie de obligaciones para el Estado y para terceros responsables promovió un cambio de escenario en términos de las políticas sociales, al incorporar estándares de derechos humanos y poner un cuño en la rutinización de los programas focalizados como única herramienta de intervención pública.
En tercer lugar, y en base al enfoque de derechos humanos, rápidamente fue apropiado por sus titulares pero también por las organizaciones de la sociedad civil para impulsar una agenda integral que se fue discutiendo en las sucesivas Conferencias Regionales de la Mujer de América Latina y el Caribe ${ }^{(3)}$, que nuclean a los gobiernos, los mecanismos para el adelanto de la mujer (MAM), las organizaciones de mujeres y de la sociedad civil junto con los organismos especializados del sistema de Naciones Unidas y del Sistema Interamericano de Derechos Humanos. Fue precisamente allí, en la Conferencia de Quito de 2007 que se comenzó por reconocer que existe un "derecho a cuidar, a ser cuidado y al autocuidado" (Pautassi, 2007) pasando a reafirmar no sólo su relevancia, sino las obligaciones positivas y negativas que implica y que exhorta en su cumplimiento a los Estados.

Las Conferencias subsiguientes celebradas en Brasilia (2010), República Dominicana (2013) y Uruguay (2016) reafirmaron en los consensos que el cuidado es un derecho y ampliaron las bases para el diseño de sistemas de provisión de cuidado basado en derechos. El Consenso de Brasilia reconoce: "Que el acceso a la justicia es fundamental para garantizar el carácter indivisible e integral de los derechos humanos, incluido el derecho al cuidado. Señalando que el derecho al cuidado es universal y requiere medidas sólidas para lograr su efectiva materialización y la corresponsabilidad por parte de toda la sociedad, el Estado y el sector privado". En la Estrategia de Montevideo (2016) se identifica a la división sexual del trabajo y la injusta organización social del cuidado como uno de los nudos estructurales a modificar de manera explícita para alcanzar sociedades más justas y sostenibles y "cerrar la brecha entre la igualdad de jure y de facto mediante el fortalecimiento de las políticas públicaspara garantizar la autonomía y el ejercicio pleno de los derechos humanos

(3) América Latina y el Caribe es la única región del mundo que se reúne sistemáticamente desde 40 años para debatir temas de género, promover consensos y acompañar la toma de decisiones para la promoción y ejercicio de la autonomía de las mujeres en el marco de la Conferencia de la Mujer. La Secretaría Técnica de la Conferencia la ejerce la División de Asuntos de Género de la Comisión Económica para América Latina y el Caribe, CEPAL, véase en https://www.cepal.org/es/areas-de-trabajo/asuntos-de-genero 
de todas las mujeres y niñas, superando discriminaciones, prejuicios y resistencias" (CEPAL, 2016).

A estos acuerdos, se suma la Convención Interamericana sobre la Protección de los Derechos Humanos de las Personas Adultas Mayores, sancionada por la Asamblea General de la Organización de Estados Americanos (OEA) en 2015, que define la obligación de los Estados de implementar medidas tendientes al desarrollo de un sistema integral de cuidado para éste grupo etáreo, estableciendo en el artículo 12 que las personas adultas tienen "derecho a un sistema integral de cuidados que provea la protección y promoción de la salud, cobertura de servicios sociales, seguridad alimentaria y nutricional, agua, vestuario y vivienda; promoviendo que la persona mayor pueda decidir permanecer en su hogar y mantener su independencia y autonomía", convirtiéndose en el primer instrumento de derechos humanos que introduce como denominación el derecho al cuidado.

Finalmente, la OIT (2019) ha establecido que el trabajo de cuidados es una prioridad como consecuencia de la adopción del Objetivo de Desarrollo Sostenible (ODS) 5, reconociendo y valorando el trabajo de cuidados no remunerado, proponiendo como vías de satisfacción la prestación de servicios públicos, infraestructuras y políticas de protección social.

En suma, el cuidado es un derecho humano que ha sido reconocido tanto respecto de su impacto narrativo y político, pero se presenta debilitado en el momento de efectivizarlo en diseños institucionales que contribuyan a garantizarlo.

A su vez, en el caso del Estado Plurinacional de Bolivia y de Ecuador se ha reconocido constitucionalmente, en 2008 y 2009 respectivamente. La ciudad de México lo incluyó en su reforma constitucional en 2017 como derecho. En agosto de 2020, la Corte Constitucional de Ecuador reconoció el cuidado como derecho y amplía las lógicas de reconocimiento entre lo formal y lo informal(4).

Por otro parte, a nivel de diseño institucional existen experiencias altamente exitosas como el Sistema Nacional de Cuidados de Uruguay, que se crea en 2010 (Batthyany, 2015) o el Sistema Nacional del Cuido de Costa Rica (Guzmán León, 2014) que se asientan en el reconocimiento del cuidado como derecho, e instalando una agenda institucional sumamente necesaria y como tercer componente en los sistemas de seguridad social. Paradojalmente en el caso de Uruguay, tras el cambio de gestión presidencial, se ha comenzado a cuestionar su relevancia, señalando que se trata de un modelo para países ricos, y en medio de la pandemia se ha comenzado con una quita presupuestaria y de prioridades. En el caso de Argentina, en diciembre de 2019 se creó el Ministerio de las Mujeres,
Géneros y Diversidades, creando una Mesa interinstitucional de cuidados y que reconoce que el cuidado es una "necesidad, un trabajo y un derecho" (MMG y D, 2020).

Si bien este cambio de su invisibilización al reconocimiento del cuidado como trabajo, luego como derecho humano, es un paso trascendental, aún resulta necesario avanzar en su efectivización y en garantías protectorias al respecto. Las razones que lo evidencian refieren al hecho que los varones no asumen plenamente sus responsabilidades respecto a sus hijas e hijos o a sus progenitores, las empresas y los empleadores en general no cumplen con sus obligaciones en relación con proveer de tiempo, dinero e infraestructura para asumir los cuidados y el Estado no universaliza las prestaciones. De esta forma, el cuidado se traslada como trabajo y como responsabilidad en las mujeres y eventualmente a nivel comunitario donde también es asumido por mujeres. A su vez, la evidencia empírica disponible en América Latina muestra, que la forma de resolver los cuidados depende de la disponibilidad de ingresos: aquellos hogares de mayor capacidad económica disponen de soluciones remuneradas, mientras que los sectores en condiciones de vulnerabilidad se encuentran limitados en su capacidad de dar respuestas y se concentran en el trabajo de las mujeres.

\subsection{La crisis preexistente}

Las medidas adoptadas durante la pandemia del Covid-19 han colocado a los cuidados como centro de una estrategia comunicativa masiva en medio de la crisis sanitaria donde el concepto de cuidado aparece de manera recurrente, tanto en la interacción cotidiana de las personas como en las acciones estratégicas oficiales. El carácter polisémico del cuidado, de los cuidados nunca antes se hizo tan claro, en la medida que cada estrategia refiere a múltiples actividades prescritas por autoridades sanitarias, máximos responsables gubernamentales y sociales, líderes religiosos y la población en general. Todas y todos hablamos de cuidados

(4) Caso 3-19-JP y acumulados. Revisión de garantías (JP) Derechos de las mujeres embarazadas y en periodo de lactancia, Corte Constitucional del Ecuador. Quito, Ecuador 5 de agosto de 2020. Véase en http://www.funcionjudicial.gob.ec/www/pdf/sentencia\%20 CC\%203-19-JP\%20y\%20acumulados.pdf 
y a cada una y cada uno nos piden que nos cuidemos, pero ¿qué significa? ¿qué diferencia hay entre la prescripción de lavarse las manos a la conformación de una forma de organización societal del cuidado, por cierto injusta, hasta invocar el derecho al cuidado? Y por último, ¿cómo se acciona en una crisis de semejante dimensión con respuestas universales e integrales?

Estos interrogantes, lejos de ser novedosos, han motivado una importante tradición de trabajos teóricos y empíricos, con abordajes interdisciplinarios que se han desarrollado centralmente desde la teoría feminista contemporánea y los estudios de género(5). Sin pretender desplegar aquí esta importantísima producción conceptual, tanto a nivel internacional como regional. Los estudios disponibles dan cuenta de qué manera a lo largo de la historia la carga de trabajo que implica la reproducción de la vida cotidiana ha sido delegada en las mujeres, desarrollando las autoras anglosajonas el concepto de care (Tronto, 2006; Fraser, 2016; Folbre, 2001), complementados por desarrollos iberoamericanos que han consolidado marcos interpretativos comunes (Borderías \& Carrasco, 1994; Torns, 2007) junto con el análisis del impacto económico del cuidado, gracias al sustantivo aporte de las economistas feministas (Picchio, 1991; Rodríguez Enríquez, 2012), Ilevaron a la redefinición del trabajo y de las relaciones que lo atraviesan. Adicionalmente, los estudios empíricos que permitieron cuantificar y calificar estas relaciones de discriminación y subordinación de las mujeres, que fueron introduciendo cambios de agenda pública relevantes, como la necesidad de medir el uso del tiempo y diseñar herramientas estadísticas adecuadas (Aguirre et al, 2005; Durán, 2012), la inclusión en las cuentas nacionales (Durán \& Diez, 2013).

Uno de los aportes sustantivos al respecto, es haber cuantificado el valor económico del trabajo de cuidados no remunerado que realizan las mujeres. De esta forma, un estudio reciente señala que el trabajo que realizan las mujeres de 15 o más años alcanza (a nivel mundial) al menos a 10,8 billones de dólares anuales, cifra que triplica el tamaño de la industria mundial de la tecnología y que está subvaluada puesto que los datos disponibles se basan en el salario mínimo y no en el salario justo (Oxfam, 2020). Estos cálculos, que a su vez se han realizado en distintos países de América Latina (Chile, México y Uruguay entre otros), muestran resultados similares comparando el aporte con sectores económicos prevalecientes en ellos.

Los estudios también han determinado la vinculación entre cuidados y migraciones en las configuraciones de las cadenas globales de cuidados (Pérez Orozco, 2007), la promoción de políticas públicas (Rico \& Robles, 2016;
Rodriguez \& Pautassi, 2014), reformas en los marcos regulatorios del servicio doméstico remunerado (Gherardi \& Durán, 2013) y la evidencia del ámbito comunitario dando respuestas a distintas demandas de cuidado (Pautassi \}\& Zibecchi, 2010) sumado al trabajo no remunerado en salud que se desarrolla en los hogares (Balardini et al., 2020). Aristas que integran el "diamante del cuidado y del bienestar" (Razavi, 1997) para referir a los cuatro actores fundamentales en la organización social del cuidado: el Estado (en tanto regulador de las relaciones sociales y proveedor de servicios); el mercado (de empleo y también como proveedor de servicios privados de cuidado tales como servicios de salud, transporte, cuidadoras y cuidadores privados); las familias (donde recae actualmente la mayor carga de las responsabilidades de cuidado, con las diferencias de género señaladas); y la comunidad (incluyendo actores como los sindicatos, cooperativas, organizaciones no gubernamentales y organizaciones barriales).

Las cuatro aristas del diamante a su vez requieren que se garantice tiempo para cuidar, dinero e infraestructura y servicios para asumirlo (Ellingstaeter, 1999), promoviendo de esta manera un efecto "desfamiliarizador" adoptado por los distintos tipos de regímenes de bienestar (Esping Anderen, 2000).

Cabe destacar que los estudios también han identificado, de manera temprana, el concepto de crisis vinculado al cuidado y la consideración del riesgo asociado al mismo. En el caso de la bibliografía anglosajona, reconoce la crisis (care crunch) asociada a la transición demográfica acelerada, aumento de la esperanza de vida, lo cual implica un constante incremento en la necesidad de cuidado prolongado en salud, así como una baja en la disponibilidad de cuidadores/as no remunerados por su ingreso al mercado de trabajo, y sin personas disponibles en los hogares para asumirlo (Lundsgaard, 2005, p. 32 ). Esta crisis se asocia centralmente a los cuidados de personas mayores, en especial en términos de trabajo no remunerado en

(5) En ONU-Mujeres (2018) se sintetizan los debates iberoamericanos en torno al cuidado, y en Pautassi y Zibecchi (2013) se concentra una mirada regional. 
salud, diferenciando cuidados a personas con diversos grados de dependencia o enfermedades en el campo de la salud mental.

En paralelo identifican las transformaciones en las familias, las que decrecen en tamaño o se fragmentan, sumada la reestructuración o precarización del empleo, el crecimiento de trabajos de tiempo parcial cuya remuneración no es suficiente para costear los gastos del hogar, incluido el cuidado, y cuya consecuencia es que se dificulte sostener a sus miembros durante sus trayectorias personales y laborales. A ello se agrega una mayor expectativa por parte de los Estados respecto a que las familias o de la comunidad van a asumir los cuidados (Hancock, 2002, p. 125). Así se conforma una suerte de "círculo de delegación" en donde todo empieza y termina en las mujeres, sin ninguna preocupación por parte de los varones respecto a esta sobrecarga.

En términos de Fraser, se están "exprimiendo" una serie de capacidades sociales claves como la disponibilidad para tener y criar niños y niñas, cuidar amigos o familiares, sostener hogares, comunidades y conformar redes, donde los problemas en el cuidado no son accidentales, sino que tienen raíces sistémicas profundas en la estructura de nuestro actual orden social (2016, p. 99). Agrega la autora que el capitalismo financiero no sólo ha disminuido la provisión pública de cuidados necesaria para la reproducción social y ha reclutado a las mujeres en el mundo asalariado, sino que además ha reducido los salarios reales, elevando por ende la cantidad de horas de trabajo remunerado de las personas en el hogar que se necesitan para mantenerlo, dando lugar a una crisis en la provisión de cuidado al interior de los hogares (Fraser, 2016, p. 114).

En el caso de América Latina, la denominada "crisis de los cuidados" (CEPAL, 2013; Rico, 2011) ha sido situada en el contexto de cambios demográficos acelerados en los países, especialmente caracterizados por el envejecimiento progresivo de las sociedades y las transformaciones producidas en los mercados de trabajo, donde la participación de las mujeres se acrecienta, atravesada por situaciones de desigualdad y discriminaciones múltiples, y donde las transformaciones en la organización familiar y social de los cuidados no se han producido, manteniéndose los varones la margen de sus responsabilidades de cuidado al tiempo que aumenta la población demandante (Rico, 2011). La ausencia de división sexual del trabajo y de los cuidados va a impactar en las trayectorias laborales y políticas de las mujeres (Rulli, 2020) como también en las responsabilidades urbanas en torno al cuidado, evidenciando las vinculaciones entre transporte, servicios públicos y cuidados (Rico \& Segovia, 2017) incluyendo también los marcos financieros internacionales y el endeudamiento externo (Bohoslavsky, 2018)(6).

\subsection{La prevención es una política pública}

Las respuestas institucionales a la situación antes descrita no asumen la crisis de los cuidados y tampoco se han responsabilizado por ella. En general, en América Latina no se han definido políticas públicas integrales, como tampoco se han producido cambios relevantes en la organización familiar del cuidado, donde los varones siguen sin realizar el trabajo de cuidados de manera activa. El hecho de que los varones han aumentado su dedicación a sus hijas e hijos y realizan tareas que antes no realizaban, no significa que hayan asumido la totalidad de sus responsabilidades. Inclusive, durante la pandemia, lo que se evidencia es, que aun visibilizando la enorme carga de trabajo que hay en los hogares, no se ha avanzado sustantivamente en su distribución.

Este avance en el reconocimiento y en las políticas públicas para su implementación posiciona sin lugar a dudas de manera diferenciada la solución durante la crisis del Covid-19. Resulta interesante señalar que, al inicio de la pandemia, entre los países que encontraron las reacciones más rápidas e integrales y con mejores soluciones para enfrentar el virus fueron aquellos cuyas gobernantes son mujeres ${ }^{(7)}$.

Por otra parte. si la recomendación que se ha lanzado es \#quedateencasa, se trata de analizar las condiciones en que \#mequedoencasa. $\mathrm{Ni}$ el cuidado para otras y otros como el autocuidado no se ejercen de manera independiente a las garantías respecto del derecho a cuidar y a ser cuidado(s) ni respecto de los demás derechos.

Tal como la Plataforma y Programa de acción de la Conferencia de Derechos Humanos de Viena (1993) en su artículo 5 establece: "Todos los derechos humanos

(6) Bohoslavsky y Rulli (2020) agregan que la androginia dominante en los organismos internacionales de asistencia crediticia es severa e identifican como una de las políticas de género más patriarcal que han impulsado es el desaliento a que los Estados inviertan fuertemente y de manera sustentable en la economía de cuidados.

(7) Se trata de países como Alemania, Dinamarca, Islandia o Nueva Zelanda entre otros. https://news.un.org/es/story/2020/05/1474002 
son universales, indivisibles e interdependientes y están relacionados entre sí. La comunidad internacional debe tratar los derechos humanos en forma global y de manera justa y equitativa, en pie de igualdad y dándoles a todos el mismo peso. Debe tenerse en cuenta la importancia de las particularidades nacionales y regionales, así como de los diversos patrimonios históricos, culturales y religiosos, pero los Estados tienen el deber, sean cuales fueren sus sistemas políticos, económicos y culturales, de promover y proteger todos los derechos humanos y las libertades fundamentales". Así el derecho al cuidado integra el conjunto de derechos que deben ser garantizados, pero también deben ser efectivizados y la pandemia es un momento donde la emergencia no es argumento válido para no garantizarlo.

En efecto, la crisis sanitaria disparada por la pandemia del COVID-19 se solapó a la crisis del cuidado preexistente con un dato aún más alarmante, ya que todo se concentra en el hogar debido a las medidas de aislamiento dispuestas, pero con un refuerzo de concentración de su resolución en las mujeres y sin un desarrollo de mecanismos institucionales para afrontarlo, acentuando las debilitadas capacidades de las mujeres para asumir tamaña crisis.

Tal como había advertido Tronto (2020) "el riesgo aparecería distinto en una sociedad que estuviera organizada en torno a la referencia al cuidado". Y desarrolla la autora el concepto de sociedad del cuidado en contraposición a la sociedad del riesgo, la cual requiere para su conformación alcanzar un equilibrio entre necesidades y los derechos como parte sustantiva de la discusión política.

En efecto, el escenario regional presenta una oportunidad, debido a su relevancia en la prevención del contagio, para avanzar en firme en la efectivización del derecho al cuidado, que incluyen de manera indisoluble políticas públicas. Ese puente entre derechos y políticas públicas, que el enfoque de derechos ha construido, es la herramienta fundamental para promover una adopción de medidas estables, intersectoriales, pero de corte institucional, donde anclar las prestaciones y acciones estatales. Tal como ha mostrado la experiencia, las crisis se resuelven mejor en el marco de las garantías de derechos humanos, siendo las políticas sociales las principales medidas de prevención. En lo que sigue, se analizará, algunas de las estrategias del cuidado durante la emergencia del Covid-19.

\section{Cuidados y emergencia: la universalidad como respuesta}

El desafío actual que la pandemia movilizó, al universalizar la estrategia de los cuidados, es precisamente la incorporación y fundamentación en el derecho al cuidado ya que a pesar de su reconocimiento aún no se considera como tal y muchos de los organismos de derechos humanos tampoco lo incorporan. En el caso de la pandemia del Covid-19, las Directrices de la Oficina del Alto Comisionado de Naciones Unidas para Derechos Humanos (2020) establecen estándares precisos y mandatos concretos a los Estados respecto al tratamiento de la pandemia y consiguientes medidas a adoptar desde un enfoque de derechos. Encabezan las recomendaciones un señalamiento de la Alta Comisionada de Naciones Unidas para los Derechos Humanos que manifiesta: “(...) nuestros esfuerzos para combatir el virus no darán resultados a menos que apliquemos un enfoque holístico, lo que significa que debemos proteger cuidadosamente a los sectores más vulnerables y desfavorecidos de la sociedad, tanto en términos médicos como económicos"(8).

La particularidad de las directrices es que establecen, al modo usual del sistema de protección universal, las prioridades de acciones respecto a los grupos vulnerables, con la novedad que en primer lugar se encuentran los "héroes y heroínas de la salud". Al respecto se destaca que las trabajadoras y trabajadores de la salud, con una sobrerrepresentación de mujeres a nivel mundial de un $70 \%$ en este sector esencial, deben recibir una remuneración adecuada y se deben contemplar las responsabilidades de cuidado propias que enfrentan en sus hogares, las que sobrecargan aún más su trabajo. Así las Directrices enfatizan que ésta situación debería ser el punto de máxima preocupación para los Estados. Si bien el reconocimiento de un sector estratégico y esencial durante la crisis es fundamental, particularmente porque se destaca la composición feminizada sectorial y las consiguientes responsabilidades asociadas al cuidado, instando a los Estados a transformar el reconocimiento en mejores remuneraciones y condiciones laborales; sin embargo, luego las directrices de Naciones Unidas no profundizan respecto de la

(8) Michelle Bachelet, Alta Comisionada de las Naciones Unidas para los Derechos Humanos (OACNUDH, 2020). 
necesidad de garantizar el derecho al cuidado de manera integral.

Continúan las directrices con las definiciones habituales de los grupos en condiciones de vulnerabilidad, estableciendo como mandato el abordaje desde un enfoque de género, recomendando la adopción de medidas específicas para reducir el impacto de la crisis sobre las mujeres, debido al trabajo de cuidado y porque asumen la mayor cantidad de tareas domésticas, con afectación sobre la salud y el empleo, debido a los mayores niveles de informalidad laboral y exposición al contagio y al despido, la falta de espacios institucionales de cuidados. Agrega la especial mirada que se debe tener sobre el impacto diferencial del Covid-19 en las personas en condiciones de pobreza, en particular respecto de las mujeres y niñas, niños y adolescentes quienes resultan los más afectados, a lo cual se debe agregar el aumento de las situaciones de violencia. Pero, nuevamente las Directrices no avanzan sustantivamente en la responsabilidad de los varones y de toda la sociedad sobre el cuidado y los cuidados. Se alude de manera global a que "entre todos nos cuidamos" pero sin destacar las responsabilidades concretas de los varones, que a lo largo de la historia han delegado las suyas en las mujeres. La oportunidad de esta crisis tan profunda habilita precisamente la posibilidad de avanzar en marcos más equitativos.

Valga como ejemplo, la situación de las cuidadoras domiciliarias de personas mayores que si bien desarrollan tareas consideradas como esenciales, no gozan de protección laboral, acceso a la salud y tampoco se las considera como heroínas. Como ejemplo, en Bolivia el caso 0 de contagio fue una cuidadora migrante de una persona mayor en Italia, que fallece como consecuencia del Covid-19 y la trabajadora regresa a su país y junto con ella ingresa el virus y la cadena de contagios ${ }^{(9)}$. Es "el caso" paradigmático de las cadenas globales de cuidados, pero que no se conoce como tal, dada la precariedad de este tipo de tareas y con todo el estigma correspondiente $^{(10)}$.

En el caso del recurso humano afectado a la salud, se estima que hay más de 3.000 trabajadores y trabajadoras de la salud han perdido la vida en el marco de la pandemia (Amnistía Internacional, 2000). Pero, además de ello, las condiciones en las que desempeñan sus actividades son de alta preocupación, que incluyen situaciones de inseguridad, despidos, violencias de todo tipo, sumado a represalias de las autoridades nacionales sanitarias y empleadores diversos. Entre las recomendaciones del informe señala que los Estados deberán reconocer al Covid-19 como enfermedad profesional con las consiguientes compensaciones monetarias, de atención médica y "otros cuidados necesarios" (Amnistía Internacional, 2020).

A nivel regional, la Comisión Interamericana de Derechos Humanos (CIDH) dictó la Resolución 1/2020, que señala de manera enfática que "si bien existen impactos sobre todos los derechos humanos frente a los diversos contextos ocasionados por la pandemia, especialmente en relación al derecho a la vida, la salud e integridad personal, se ven seriamente afectados el derecho al trabajo, a la seguridad social, a la educación, a la alimentación, al agua y a la vivienda, entre otros DESCA" (CIDH, 2020, p. 5) y luego enfatiza centralmente los deberes de protección respecto al derecho a la salud. Al momento de recomendar acciones vinculadas al cuidado, si bien explícitamente advierte que en general los cuidados recaen centralmente sobre las mujeres a expensas de su desarrollo personal y laboral, agrega la Comisión que los cuidados durante la pandemia son mayores y más exigentes, y se cuenta con un escaso grado de institucionalización y falta de reconocimiento social o económico. Pero en el momento de las recomendaciones se concentra en los grupos históricamente excluidos.

En términos de recomendaciones de acciones y políticas, la CIDH (2020) insta a que, en el momento de adoptar cualquier medida durante la pandemia, las mismas deben incluir un enfoque interseccional, de género, cultural y de derechos humanos, garantizando un tratamiento especial y con mayor urgencia respecto a los grupos vulnerables o históricamente excluidos. En especial, la recomendación se concentra respecto de "personas mayores y personas de cualquier edad que tienen afecciones médicas preexistentes, personas privadas de libertad, mujeres, pueblos indígenas, personas en

(9) La prensa boliviana recoge dos casos 0 paralelos, una mujer migrante de Santa Cruz de la Sierra, que era cuidadora, y otra mujer en Oruro que arriban ambas en el mismo vuelo de Italia. Véase en https://www.atb.com.bo/sociedad/una-mujer-de-60-a\%C3\%B1osque-lleg\%C3\%B3-de-italia-es-el-primer-caso-de-coronavirus-en-santa-cruz. En el primer caso, felizmente se recupera tras un largo período de internación, véase en https://www.opinion.com.bo/articulo/pais/paciente-cero-sale-airosa-55-dias-convierte-simboloesperanza/20200506022254766062.html.

(10) Flavia Marco Navarro, Plataforma de Corresponsabilidad Social y Publica del Cuidado, Bolivia; comunicación personal agosto 2020. 
situación de movilidad humana, niñas, niños y adolescentes, personas LGBTI, personas afrodescendientes, personas con discapacidad, personas trabajadoras, y personas que viven en pobreza y pobreza extrema, especialmente personas trabajadoras informales y personas en situación de calle; así como en las defensoras y defensores de derechos humanos, líderes sociales, profesionales de la salud y periodistas".

Si bien a priori el acuerdo es inmediato sobre esta recomendación protectoria y la necesaria prioridad, al igual que en el campo del cuidado y de los estudios de género existe una importantísima producción académica sobre grupos desaventajados. Al respecto, debido a que los ideales de derechos igualitarios han sido sistemáticamente apropiados y solo ejercidos para determinados sectores de poder que han utilizado este principio igualitario para su propio beneficio, diversas corrientes defienden abandonar de la idea de "derechos iguales para todos" ya que en los hechos no funcionan como tales en el momento de hacerlos efectivos, para pasar al otorgamiento de "derechos especiales" para ciertos grupos, que se encuentran bajo subordinación perpetua, los que intencionadamente o no reciben tratamiento arbitrario (Fiss, 1999; Gargarella, 1999). Las medidas de acción afirmativa y las cuotas son medidas similares que han sido centrales para garantizar el acceso de mujeres en el mundo del trabajo remunerado y en puestos de participación política. Es decir, la utilidad de estos argumentos y acciones es indiscutible para garantizar la efectivización de derechos.

Sin embargo, hay ciertas particularidades que la consideración de las mujeres como grupo escapa a la verificación empírica. En primer lugar, por ser más de la mitad de la población y porque precisamente la heterogeneidad caracteriza a su situación, debido a la forma que adquiere la estructura patriarcal en nuestras sociedades, opera a que el atravesamiento de las vulnerabilidades impacte sobre todas ellas, independientemente de la posición social, económica o nivel educativo alcanzado. $Y$ si bien han manifestaciones diferenciales, la violencia doméstica es un indicador contrafáctico que atraviesa a todas las mujeres, inclusive las de las clases sociales más acomodadas (Gherardi, 2016).

Es decir, busco destacar que si no se considera la integralidad protectoria del cuidado se está desaprovechando una oportunidad para implementarlo en su carácter de derecho humano y cuando se recomiendan medidas nuevamente se concentra en los "grupos" y dentro de ellas en las mujeres ${ }^{(11)}$. Es decir, si bien en los hechos son las mujeres quienes asumen el cuidado, no se repara que la crisis es el momento para avanzar en su distribución, interpelando las responsabilidades de los varones y de la sociedad en su conjunto. Los varones tienen la misma responsabilidad de cuidado solo que no la han asumido y por sí solos, salvo excepciones, no están dispuestos a hacerlo.

Si bien es cierto que, durante la crisis, la urgencia de las reacciones de los mecanismos protectorios de derechos humanos antecede las reflexiones conceptuales innovadoras, el enfoque de derechos humanos debe concentrarse en estándares universales y concretos de intervención. Es decir, no es la vulnerabilidad de la crisis lo que debe activar las respuestas, sino el solo hecho de ser persona. Por otra parte, se repara a su vez muy poco en quienes deben recibir cuidados y respecto al autocuidado las referencias son aún más escasas como derecho. Sin embargo, existe un traslado de responsabilidades a que cada individuo se cuide, y mucho, a sí mismo. Pero quien va a proveer las precondiciones para que esa persona se cuide son las mujeres en los hogares. La pregunta es ¿hasta cuándo van a poder sostener las mujeres tanta sobrecarga de trabajo y responsabilidades? Pero aún más grave, ¿a nadie le parece mal? ¿cómo se evalúa de cara al principio de igualdad?

Valga como ejemplo que durante la pandemia, las recomendaciones a los Estados de garantizar una vida libre de violencias para mujeres e identidades sexuales y actuar de manera preventiva al respecto fueron inmediatas. De esta manera, el Comité de Expertas en Violencia (CEVI) para el seguimiento de la Convención de Belem do Pará (MESECVI, 2020) fue uno de los primeros en advertir la propagación de las violencias en un doble sentido: tanto respecto al ámbito público como privado. Así las expertas integrantes del Comité destacaron que la cuarentena obliga a las mujeres a convivir con sus agresores y que en la medida que se mantenga el aislamiento, los Estados deben adoptar acciones directas y específicas para prevenir la violencia, ya que de lo contrario va a seguir en aumento. $Y$

(11) Al respecto, los argumentos de Fineman (2013) respecto a los límites del concepto de vulnerabilidad y la necesaria consideración de la potencialidad de que todas las personas somos vulnerables cuestionando las respuestas asistenciales y excluyentes. Por su parte MacKinnon (1991) enfatiza la necesidad de sustituir el concepto de discriminación por el de dominación. 
de hecho ha sucedido, con impacto respecto a las relaciones intrafamiliares. UNICEF ha señalado que "Las niñas y niños son las víctimas ocultas del coronavirus" (2020, p. 6).

En efecto, se ha alertado en relación con las secuelas psicológicas y emocionales que el aislamiento obligatorio produce, desde situaciones de depresión y angustias en adolescentes a los riesgos que corren NNA por el maltrato y violencia al interior de los hogares. UNICEF (2020) recomienda reforzar los mecanismos de atención y denuncia, pero en particular respuestas rápidas y seguimiento cercano de los casos de violencia anteriores a la pandemia o durante, de modo de evitar situaciones de agudización. Y podríamos seguir enumerando las recomendaciones respecto a pueblos indígenas, migrantes, personas con discapacidad, colectivos LGTBI y diversidades sexuales. Nuevamente si bien la especificidad debe ser incorporada en su totalidad y el respeto de las particularidades de cada persona, ello no obsta a la aplicación del estándar de universalidad.

Asimismo, la crisis del Covid-19 también ha permitido visualizar las condiciones de inserción laboral de un importante número de personas en América Latina. Particularmente se han evidenciado los altos índices de precariedad e informalidad laboral, que concentra una de las principales formas de inserción laboral de las mujeres que alcanza al $54,3 \%$ de ellas (Organización Internacional del Trabajo, 2018). De manera reciente la OIT (2020) ha señalado que los sectores de servicios son los más afectados durante la crisis, y donde se han producido el mayor cierre de establecimientos junto con el cierre de mercados callejeros, la prohibición y persecución del comercio ambulante, que en estos últimos casos es donde trabajan la mayoría de las mujeres, ya que les permite asumir el cuidado de sus hijas e hijos (Alfers, Moussié \& Harvey, 2020).

Y claro está, quienes resultan más afectadas son las diez millones de trabajadoras domésticas remuneradas de América Latina, muchas de ellas migrantes que no tienen donde ir y tampoco pueden regresar a sus países, quienes a la vez que realizan quehaceres del hogar, suelen hacerse cargo de parte importante del cuidado directo. En situación similar están las cuidadoras a domicilio, que en general se ocupan de personas mayores. Las asalariadas de este sector de la economía se han visto profundamente afectadas por despidos, retrasos en el pago o se les han quitado sus salarios, además de una exposición al virus por falta de medidas de bioseguridad en muchos de los hogares. También se enfrentan con presiones para continuar trabajando a pesar de las normas de cuarentena y prohibición de circulación, debido a que las y los empleadores argumentan que deben realizar teletrabajo (jerarquizando su tarea) y requieren que se asuman las tareas de limpieza y de cuidado, en especial de las niñas y niños en el hogar.

Respecto a este último punto, aquellas trabajadoras que ejercen cargos profesionales y poseían acceso a la tecnología, han sido destinadas a sus viviendas para efectuar teletrabajo con dedicación y jornada definida, y en muchos casos, a la vez que cuidan y educan a hijas e hijos que no están asistiendo a la escuela. También asumen la realización de tareas de limpieza y domésticas habituales sumadas a las de higiene directamente vinculadas con la prevención del contagio por Covid-19, sumado a la atención de personas mayores que están bajos protocolos más estrictos y restricciones de movilidad por ser población en riesgo frente a la enfermedad.

Esta "conciliación", que ya era limitada anteriormente en los espacios habituales de trabajo, prácticamente desaparece en la cuarentena, sin horarios ni límites en la demanda de las y los empleadores, sin pensar en las dificultades para montar una "oficina en casa" junto con la "escuela" en casa, con los problemas asociados, sin espacio físico, mobiliario ni conexión adecuada a internet. En los países de la región, en general no se ha legislado sobre teletrabajo o modalidades similares, y en los casos que la pandemia movilizó la presentación de proyectos legislativos, no contemplan en general el ejercicio del derecho al cuidado ${ }^{(12)}$. Por otra parte, las reivindicaciones de las y los trabajadores es a ejercer el "derecho a la desconexión" dada las presiones patronales. Estas y otras situaciones demandan una revisión respecto que el traslado de los espacios laborales al hogar no se puede producir de manera automática y sin costos sobre las personas y sus familias, en especial sobre las familias.

Adicionalmente, las demandas de cuidado son más intensas para los hogares de menos ingresos, que se caracterizan por ser hogares compuestos por parejas con hijos e hijas, o que están a cargo de una mujer con hijos e hijas, o de hogares extendidos. Los hogares del quintil más bajo de ingresos cuentan claramente

(12) Es el caso de Argentina que sancionó en agosto de 2020 la Ley 27555 que regula el contrato de teletrabajo incluyendo explícitamente el derecho a la desconexión y el reconocimiento de las tareas de cuidados (artículo 6). 
con menores recursos y oportunidades para enfrentar y asumir estas demandas de cuidado. Ante una limitada oferta pública de servicios e infraestructura de cuidado (pocos espacios de cuidado infantil, jornadas escolares simples e incompatibles con jornadas laborales, horarios acotados para la atención de la salud en ámbitos públicos, un sistema de transporte que no responde a las necesidades de las familias), sólo aquellas las familias que pueden asumir esos costos, adquieren servicios para el cuidado en el mercado. Así, las familias de ingresos más altos contratan tales servicios bajo la forma de escolaridad, transporte o servicios de salud privados, o la contratación de trabajadoras del hogar(13). De allí que las medidas que, en algunos países, como Argentina, implementaron transferencias monetarias de emergencia de manera ágil apenas declarada la pandemia fueron sumamente eficaces para contener la crisis de ingresos (Arcidiácono \& Gamallo, 2020). Si se analizan los datos comparados provistos por el Observatorio Covid 19 de la CEPAL, el $78 \%$ de los países incorporó nuevas transferencias monetarias en efectivo y en un $69 \%$ lo hizo en alimentos o en prestaciones en especie, mientras que ha garantizado los servicios básicos en un $72 \%$ (CEPAL, 2020).

Claramente durante la pandemia y el aislamiento preventivo el núcleo duro de desigualdad respecto del tiempo y las tareas se incrementa, sin signos de alarma por parte de los integrantes del hogar, pero sí con síntomas concretos de deterioro en la salud de las mujeres, con incrementos en los niveles de angustias y estrés emocional, sin tiempo para descanso y tareas recreativas.

La evidencia empírica relevada, por cierto, escasa y sin considerar muchas otras situaciones de cuidados como los demandados por las personas mayores, personas con discapacidad y enfermedades crónicas entre otras, nos señala que la falta de distribución de tareas de cuidado entre mujeres y varones se ha agravado respecto a los niveles preexistentes a la pandemia (ARF, 2020; CEPAL, 2020). No solo las medidas de aislamiento han interrumpido las redes familiares de cuidados - la presencia de abuelas, tías, vecinas o espacios comunitarios- sino que la falta de instituciones como escuelas y centros comunitarios, sumado a la falta de personal de casas particulares, han visibilizado la complejidad de los cuidados y su concentración, que en muchos casos se desarrollan en un espacio físico limitado, como son los hogares medios urbanos, y delegadas en las mujeres.

A más de seis meses de iniciadas las medidas de aislamiento en la región, con cierres totales, toque de queda, aperturas paulatinas, estrategias de alertas y semáforos en función de la movilidad de las curvas de contagio, la tendencia muestra poca variación en los próximos meses ${ }^{(14)}$. Es decir, América Latina transitará entre el confinamiento y la reapertura de las economías de manera alternada lo cual es necesario que estas situaciones se contemplen en las medidas a adoptar.

Que hoy se visibilice la enorme carga de trabajo que implican los cuidados, que se busque responder con medidas gubernamentales más amplias, con una invocación a derechos y en el marco de la urgencia, no significa que se esté distribuyendo. Lamentablemente nada es novedoso, todo estuvo antes de la irrupción del coronavirus y el cuidado, una vez más, recae sobre las mujeres, sin que siquiera alarme a las y los integrantes de su propia familia.

La oportunidad que el reconocimiento del derecho al cuidar, a ser cuidado y al autocuidado trae aparejada en el campo de la institucionalidad, es precisamente la posibilidad de diseñar nuevos arreglos institucionales que articulen sistemas que organicen las demandas crecientes y diversas de cuidado con respuestas integrales (y no feminizadas). No se trata de reconocer el cuidado que asumen las mujeres sino avanzar en su distribución.

\section{Conclusión: la integralidad de derechos en la agenda pos- pandemia}

La pandemia del coronavirus ha "atravesado" al mundo entero. De acuerdo con los postulados del enfoque de derechos humanos requiere soluciones integrales, necesariamente unidas a la aplicación del enfoque de género, que ya no solo abarca estrategias nacionales, sino globales, e involucra a todos los países. La

(13) ONU-Mujeres ha implementado una serie de acciones para garantizar un efectivo tratamiento de la crisis de los cuidados en las mujeres para América Latina y el Caribe. Véase en https://lac.unwomen.org/es/digiteca/publicaciones/2020/03/covid-como-incorporara-las-mujeres-y-la-igualdad-de-genero-en-la-gestion-de-respuesta.

(14) El Observatorio Covid 19 de CEPAL ha identificado 391 acciones desplegadas en América Latina y el Caribe que limitan o prohíben circulación entre países o al interior de los mismos, de las cuales 136 restringen o cierran lugares y espacios públicos prohíben reuniones masivas. 
propagación del virus y su rapidez son un claro ejemplo y los millones de vidas que ha cobrado alertan sobre la urgencia en dar respuestas. Del mismo modo que en cuando hubo acciones cooperativas y mancomunadas entre países y jurisdicciones las respuestas fueron más eficaces, y si además se trata de gobiernos liderados por mujeres, todavía más. Pero superado los picos, las alarmas vuelven a encenderse debido a rebrotes o nuevos casos, o mutaciones de otros virus. Una y otra vez, cuidarse es indispensable, pero está claro que como acción individual no es suficiente.

Es por ello que las acciones públicas que se adopten deben fundarse en las obligaciones positivas y negativas que corresponden al derecho al cuidado, tanto para el Estado, los mercados, las familias y las organizaciones sociales comunitarias. $Y$ dentro de las familias emergen las obligaciones que corresponden a los varones, quienes a la fecha no las han asumido. La transformación sobre las relaciones de cuidado se hace con ellos y deben ser responsabilizados por su omisión. Del mismo modo, el mercado, los Estados y las organizaciones sociales comunitarias tienen que rendir cuentas por su falta de acciones integrales y responsables en torno a los cuidados.

Lejos de construir un argumento que contraste urgencia con universalidad, protección especial versus desprotección, asistencialismo versus universalidad, economía o salud, busco advertir que uno de los escenarios pos-pandemia es precisamente repensar estos esquemas que priorizan la condición de vulnerabilidad por sobre la integridad de la persona y que descargan la solución de las crisis en las mujeres. Sin duda que la vulnerabilidad debe priorizar acciones debido a su urgencia, considerando las diferencias y particularidades, pero siempre desde el respeto a la universalidad y en una justa distribución de trabajo y de cuidados.

La base del accionar histórico de las burocracias asistenciales en América Latina es el supuesto de la elasticidad infinita del trabajo de las mujeres. De qué nueva "normalidad" podemos hablar si no se produce una ruptura de los patrones de desigualdad estructurales existentes y que la solución de la crisis se traslade a las mujeres. De la manera en que ellas han realizado sus tareas como agentes de prevención, sostén y cuidados de los integrantes de sus familias se mide el impacto positivo del aislamiento, y por lo tanto del manejo de la crisis.

La economía no monetaria de los hogares está garantizando la continuidad del aislamiento preventivo y el cuidado es central para aislar a la población y evitar contagios. Estas medidas se siguen prolongando indefinidamente en América Latina y confrontan con tensiones en torno a la apertura o las restricciones, pero lo cierto es que conocemos muy poco acerca del Covid-19. Pero lo que sí sabemos es que la responsabilidad de evitar que se siga propagando se concentre en las mujeres. Si antes de la crisis la relación era altamente inequitativa sin que a muchos les preocupara demasiado, hoy resulta insostenible y muy lejos se encuentra de pensar patrones de "nueva normalidad".

Las garantías con respecto al cuidado no pueden basarse en una supuesta neutralidad, sino que deben identificar las individualidades en un marco de responsabilidad colectiva, considerando acciones para la superación de las brechas de género hoy existentes. La salida a la pandemia es con derechos humanos, con igualdad y con claridad respecto a la necesidad de incluir el enfoque de género y diversidades. En consecuencia, no se trata de garantizar derechos "para las mujeres" sino precisamente revisar y transformar la asignación naturalizada e injusta del trabajo productivo y de cuidados en ellas.

Una de las enseñanzas centrales del aislamiento es que la falta de visibilización de la injusta división sexual y social del trabajo remunerado y de cuidado ha llegado a su techo, del mismo modo que las violencias. Tal como he desarrollado a lo largo del artículo, ya no hay vuelta atrás para identificar que el cuidado es un trabajo intenso, fundamental para la sociedad, pero también es un derecho humano que requiere ser satisfecho y que además demanda una necesaria (re) distribución.

Las propuestas están disponibles, se trata de adoptarlas, tanto a nivel estatal, empresarial, actores sociales, políticos, sindicales y particularmente a nivel familiar y social. Desde un enfoque de derechos humanos se debe transformar el riesgo actual situando a los cuidados, remunerados y no remunerados como eslabones centrales de la salida de la crisis, pero garantizando su efectiva protección y distribución.

Dado que la pandemia no se presenta como un escenario temporario y acotado, el regreso a la "normalidad" o a ciertos escenarios de la vida pre-pandemia, aun en un cálculo optimista, es posible que dure varios años. $Y$ en este período crítico, los países deberán adoptar medidas de entrada y salida del aislamiento con mayor o menor restricción en el distanciamiento social. En paralelo, deberán impulsar procesos de (re) organización de las economías hoy en crisis, de los agotados sistemas de salud y de una 
economía no monetaria en sus límites, con la marca del número dramático de víctimas, en un contexto de desigualdad agravada.

Este complejo escenario puede visualizarse como una oportunidad para promover una transformación sobre la injusta configuración de los cuidados. Pero, además, es el momento de identificar la inacción estatal y la falta de cumplimiento de sus obligaciones positivas, junto con las responsabilidades de actores económicos, sociales, sindicales y familiares. Si como personas no asumimos la responsabilidad que nos compete respecto a la sostenibilidad de la vida poco habremos aprendido. En otras palabras, es la oportunidad para cuestionar de qué normalidad hablamos, transformar el riesgo y poner a prueba la relevancia de los derechos humanos.

\section{Referencias bibliográficas}

Aguirre, R., García Sánchez, C. \& Carrasco, C. (2005). El tiempo, los tiempos, una vara de desigualdad. CEPAL, (65), 1-83.

Alfers, L., Moussié, R. \& Havey, J. (2020). The COVID-19 crisis: income support to informal workers is necessary and possible. OECD. https://oecd-development-matters.org/2020/04/22/the-covid-19-crisisincome-support-to-informal-workers-is-necessary-and-possible/

Amnistía Internacional. (2020). Expuesto, acallado y atacado. Deficiencias en la protección del personal sanitario y que realiza labores esenciales durante la pandemia del Covid-19. Amnesty International 2020, 1-63. https://www.amnistia.org.pe/descargar/ DeficienciaProteccionCovid.pdf

Articulación Regional Feminista (ARF). (2020). Los derechos de las mujeres de la región en épocas de Covid-19. Estado de situación y recomendaciones para promover políticas con justicia de género. Equipo Latinoamericano de Justicia y Género. http://www.ela.org.ar/a2/index. cfm?muestra\&aplicacion=APP187\&cnl=87\&opc=53\&codcontenido=4220\&plcontampl=43

Balardini, L., Gherardi, N., Martelotte, N. \& Pautassi, L. (2020). El trabajo no remunerado de cuidado de la salud: una mirada desde la igualdad de género. Organización Panamericana de la Salud. https:// iris.paho.org/handle/10665.2/52207

Batthyány, K. (2015). Las políticas y el cuidado en América Latina: Una mirada a las experiencias regionales. CEPAL, (124), 1-50.

Bohoslavsky, J. P. \& Rulli, M. (2020). Covid-19, instituciones financieras internacionales y continuidad de las políticas androcéntricas en América Latina. Revista Estudos Feministas, 28(2), 1-16. https://www.scielo.br/ scielo.php?script=sci arttext\&pid=S0104-026X2020000200201

Bohoslavsky, J. P. (2020). COVID-19 y Derechos Humanos. La pandemia de la desigualdad. Colección Derechos Sociales y Políticas Públicas Editorial Biblos.

Borderías, C. y Carrasco, C. (1994). Las mujeres y el trabajo: aproximaciones históricas, sociológicas y económicas. (Icaria Editorial, ed.). ICARIA-FUHEM.

Comisión Económica para América Latina y el Caribe y Organización Internacional del Trabajo. (2020). El trabajo en tiempos de pandemia: desafíos frente a la enfermedad del coronavirus (COVID-19). Coyuntura laboral en América Latina y el Caribe, (22), 1-60. https://www.cepal.org/es/publicaciones/tipo/ informe-cepal-oit

Comisión Económica para América Latina y el Caribe. (2020). Observatorio Covid-19 en América Latina y el Caribe [mapa o infografía]. Sistea de Coordinadores Residentes de naciones Unidas en América Latina y el Caribe, ONU Mujeres y CEPAL. https://www.cepal.org/es/temas/covid-19

Comisión Económica para América Latina y el Caribe. (2013). Panorama Social de América Latina y el Caribe 2013 (LC/G 2580). https://www.cepal. org/es/publicaciones/35904-panorama-socialamerica-latina-2013

Comisión Interamericana de Derechos Humanos. (2020, 10 de abril). Resolución 1/2020. Pandemia y Derechos Humanos en las Américas. Washingthon D.C. https://www.cepal.org/es/temas/covid-19

Durán, M. A. (2012). El trabajo no remunerado en la economía global. (Fundación BBVA, 1. ${ }^{\mathrm{a}}$ ed.). Rubes Editorial. http:// digital.csic.es/handle/10261/76517

Durán, M. A. y García S. (2013). Economía del Cuidado. En L. Pautassi y C. Zibecchi (coords.), Las fronteras del cuidado. Agenda, derechos e infraestructura (pp. 155-190). Editorial Biblos.

Ellingstaeter, A. (1999). Dual Breadwinners between State and Market. En R. Crompton (ed.), Restructuring gender relations and employment. The Decline of the Male Breadwinner (pp. 364-365). Oxford University Press.

England, P., Budig, M. y Folbre, N. (2002). Wages of Virtue: The Relative Pay of Care Work. Social Problems, 49(4), 455-473.

Fineman, M. A. (2013). Equality, Autonomy, and the Vulnerable Subject in Law and Politics. En M. Fineman y A. Grear (eds.), Vulnerability. Reflections on a New Ethical Foundation for Law and Politics (pp. 13-27). Ashgate Publishing. https://doi. org/10.4324/9781315547862

Fraser, N. (2016). Contradictions of Capital and Care. New Left Review, (100), 99-117. https:// newleftreview.org/issues/II100/articles/nancyfraser-contradictions-of-capital-and-care

Folbre, N. (2001). The invisible Heart. Economics and Family Values. The New York Press.

Gherardi, N. \& Durán, J. (2013). La discriminación en casa: regulación del servicio doméstico en la Argentina. En: L. Pautassi y C. Zibecchi (eds.), Las fronteras del cuidado. Agenda, derechos e infraestructura (pp. 249-272). Editorial Biblos.

Guzman, J. (2014). Red Nacional de Cuido y Desarrollo Infantil en Costa Rica. El proceso de construcción 2010-2014 (LC/L.3858). Serie Políticas Sociales. Comisión Económica para 
América Latina y el Caribe. https://www.cepal. org/es/publicaciones/36819-red-nacionalcuido-desarrollo-infantil-costa-rica-procesoconstruccion-2010-2014

Hancock, L. (2002). The care crunch: changing work, families and welfare in Australia. Critical Social Policy, 22(1), 119-140. https://doi.org/10.11 $77 / 02610183020220010101$

MacKinnon, C. (1991). Difference and Dominance: On Sex Discrimination. En K. Bartlett y R. Kennedy (eds.), Feminist Legal Theory: Readings in Law and Gender (pp. 81-94). Westview Press.

Marco, F. (2009). Legislación comparada en material de familias. Los casos de cinco países de América Latina (LC/L. 3102-P). Serie Políticas Sociales. Comisión Económica para América Latina y el Caribe. https://www.cepal.org/es/ publicaciones/6155-legislacion-comparadamateria-familias-casos-cinco-paises-americalatina

Marco F. \& Pautassi, L. (2020). La compensación del cuidado en los sistemas de pensiones en América Latina. Revue Internationales des Études du Dévelo, (242), 143-165. https://dialnet.unirioja. es/servlet/articulo?codigo $=7455383$

Organización Internacional del Trabajo. (2019). El trabajo de cuidados y los trabajadores del cuidado para un futuro con trabajo decente. https://www. ilo.org/global/publications/books/WCMS_633168/ lang--es/index.htm

ONU Mujeres. (2018). El trabajo de cuidados: una cuestión de derechos humanos y políticas públicas. https://mexico.unwomen.org/es/digiteca/ publicaciones/2018/mayo-2018/mayo/publicacionde-cuidados

OXFAM Internacional. (2020). Tiempo para el cuidado. El trabajo de cuidados y la crisis global de desigualdad. https://oxfamilibrary.openrepository. com/bitstream/handle/10546/620928/bp-time-tocare-inequality-200120-es.pdf

Pautassi, L. (2007). El cuidado como cuestión social desde un enfoque de derechos. Serie Mujer y Desarrollo, (87), Comisión Económica para América Latina y el Caribe.

Pautassi, L. (2018). El cuidado: de cuestión problematizada a derecho. Un recorrido estratégico, una agenda en construcción. En: ONU Mujeres (ed.), El trabajo de cuidados: una cuestión de derechos humanos y políticas públicas (pp. 178191). ONU Mujeres México.

Pautassi, L. (2019). La emergencia del cuidado en los ámbitos locales: múltiples configuraciones En La agenda emergente de las Políticas sociales. Movilidad urbana, cuidado y violencias de género. Editorial Biblos.
Pautassi, L. \& Zibecchi, C. (2013). Las fronteras del cuidado. Agenda, derechos e infraestructura. Editorial Biblos.

Pautassi, L. \& Zibecchi, C. (2010). La provisión de cuidado y la superación de la pobreza infantil. Programas de transferencias condicionadas en Argentina y el papel de las organizaciones sociales y comunitarias (LC/L.3198-P). Serie Políticas Sociales 159. Comisión Económica para América Latina y el Caribe. https://repositorio.cepal. org/bitstream/handle/11362/6164/1/S1000086_es.pdf

Pérez, A. (2007). Cadenas globales de cuidado [Documento de trabajo 5]. UN-INSTRAW

Picchio, A. (2001). Un enfoque macroeconómico ampliado de las condiciones de vida. En C. Carrasco (ed.), Tiempos, trabajos y género (pp. 15-40). Publicaciones de la Universitat de Barcelona. https://www. fundacionhenrydunant.org/images/stories/biblioteca/Genero-MujerDesarrollo/enfoque\%20macroeconomico\%20ampliado.pdf

Razavi, S. (2007). The Political and Social Economy of Care in a Development Context. United Nations Research Institute for Social Development (UNRISD). https://www.unrisd.org/80256B3C005BCCF9/ (httpAuxPages)/2DBE6A93350A7783C12573240036D5A0/\$file/ Razavi-paper.pdf

Rico, M. N. (2011). Crisis del cuidado y políticas públicas: el momento es ahora. Las familias latinoamericanas interrogadas: hacia la articulación del diagnóstico, la legislación y las políticas.

Rico, M. N. \& Robles, C. (2016). Políticas de cuidado en América Latina Forjando la igualdad. Serie Asuntos de Género, número 140, 1-81. https://www.cepal.org/es/publicaciones/40628-politicas-cuidadoamerica-latina-forjando-la-igualdad

Rico, M. N. y Segovia, O. (2017). ¿Quién cuida en la ciudad? Aportes para políticas urbanas de igualdad. Comisión Económica para América Latina y el Caribe. https://www.cepal.org/es/publicaciones/42424quien-cuida-la-ciudad-aportes-politicas-urbanas-igualdad

Rodríguez, C. (2012). La cuestión del cuidado: ¿El eslabón perdido del análisis económico?. Revista CEPAL, (106), 23-36. https://www.cepal. org/es/publicaciones/11524-la-cuestion-cuidado-eslabon-perdidoanalisis-economico

Rulli, M. (2020). Madres y/o políticas: Entre el derecho a participar y el derecho al cuidado. En, M. Herrera, S. Fernández, N. De la Torre \& C. Videtta (coords.), Tratado de Géneros, derechos y Justicia (1. ${ }^{\mathrm{a}}$ ed.), Editorial Rubinzal Culzoni.

Torns, T. (2007). El tiempo de trabajo y las relaciones de género: las dificultades de un cambio ineludible. En C. Prieto (ed.), Trabajo, género y tiempo (pp. 269-277). Editorial Complutense.

Tronto, J. (2006). Vicious circle of privatized caring. En M. Hamington y D. Miller (eds.), Socializing Care: Feminist Ethics and Public Issues (pp. 3-26). Rowman \& Littlefield Publishers.

Tronto, J. (2020). ¿Riesgo o cuidado? (1. ${ }^{\text {a }}$ ed.). Fundación Medifé Edita. https://issuu.com/fundacionmedife/docs/riesgo_o_cuidado-issuu

UNICEF. (2020). Encuesta de Percepción y Actitudes de la Población. Impacto de la pandemia COVID-19 y las medidas adoptadas por el gobierno sobre la vida cotidiana. UNICEF Argentina. https://www. unicef.org/argentina/media/8056/file/Covid19-EncuestaRapidaInformeEducacion.pdf 